\title{
The more the merrier? How a few SNPs predict pigmentation phenotypes in the Northern German population
}

\author{
Amke Caliebe ${ }^{1,4}$, Melanie Harder ${ }^{2,4}$, Rebecca Schuett ${ }^{2,5}$, Michael Krawczak ${ }^{1}$, Almut Nebel ${ }^{3}$ \\ and Nicole von Wurmb-Schwark ${ }^{\star, 2,6}$
}

\begin{abstract}
Human pigmentation traits are of great interest to many research areas, from ancient DNA analysis to forensic science. We developed a gene-based predictive model for pigmentation phenotypes in a realistic target population for forensic case work from Northern Germany and compared our model with those brought forth by previous studies of genetically more heterogeneous populations. In doing so, we aimed at answering the following research questions: (1) do existing models allow good prediction of high-quality phenotypes in a genetically similar albeit more homogeneous population? (2) Would a model specifically set up for the more homogeneous population perform notably better than existing models? (3) Can the number of markers included in existing models be reduced without compromising their predictive capability in the more homogenous population? We investigated the association between eye, hair and skin colour and 12 candidate single-nucleotide polymorphisms (SNPs) from six genes. Our study comprised two samples of 300 and 100 individuals from Northern Germany. SNP rs12913832 in HERC2 was found to be strongly associated with blue eye colour (odds ratio $=40.0, P<1.2 \times 10^{-4}$ ) and to yield moderate predictive power (AUC: $77 \%$; sensitivity: $90 \%$, specificity: $63 \%$, both at a 0.5 threshold for blue eye colour probability). SNP associations with hair and skin colour were weaker and genotypes less predictive. A comparison with two recently published sets of markers to predict eye and hair colour revealed that the consideration of additional SNPs with weak-to-moderate effect increased the predictive power for eye colour, but not for hair colour.
\end{abstract}

European Journal of Human Genetics (2016) 24, 739-747; doi:10.1038/ejhg.2015.167; published online 19 August 2015

\section{INTRODUCTION}

Elucidating the genetic basis of human pigmentation traits such as eye, hair and skin colour is of great interest in many areas of scientific research. For example, pigmentation traits are known to be associated with a number of human diseases, including melanoma and nonmelanoma skin cancer. ${ }^{1-3}$ Moreover, the prediction of pigmentation phenotypes from genotypes would be highly relevant to ancient DNA research ${ }^{4-6}$ and, if and when legally possible, to forensic case work, particularly for solving cases without a suspect. ${ }^{7,8}$

The aetiology of human pigmentation traits is thought to be highly complex, possibly involving gene-gene and gene-environment interactions, ${ }^{9-11}$ and a high level of phenotypic diversity has been observed among individuals of European descent. ${ }^{12,13}$ Interestingly, while the heritability of eye and hair colour is known to be very strong, skin colour appears to be less genetically determined. ${ }^{14}$

In the recent past, some important advances have been made towards the identification of genes associated with human pigmentation traits. For eye and hair colour, a few major genes show large effects even though some additional genes of minor effect have been found to be trait-associated as well in genome-wide studies, thereby highlighting the polygenic nature of these phenotypes. The HERC2/OCA2 genes have a specifically strong effect on human eye colour. ${ }^{10,15-18}$ Single-nucleotide polymorphism (SNP) rs12913832 in HERC2 shows a particularly strong genotypephenotype association that is potentially modified by SNP rs 1800407 in OCA2, ${ }^{10,17}$ a gene involved in human pigmentation via the regulation of melanin production. ${ }^{19}$ More recently, the rs 12913832 region of HERC2 has been reported to act as an enhancer of the transcription of OCA2. ${ }^{19}$

The red hair phenotype appears to be predominantly determined by the $M C 1 R$ gene, ${ }^{20}$ which encodes the melanocortin receptor. For other hair colours, such as blond, brown and black, and for skin colour, weaker genetic associations involving OCA2 and HERC2 have been described in European populations. ${ }^{10}$ Additional genes suggested to have a minor effect upon pigmentation phenotypes include

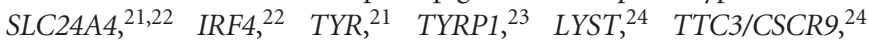
$A S I P^{23}$ and SLC45A5, ${ }^{25}$ among others.

Recently, the prediction of external features from DNA data has gained considerable interest in forensic science, where this approach is also referred to as 'forensic DNA phenotyping', and where considerable progress has been made in this regard, particularly for eye colour. ${ }^{7,26-28}$ Two marker sets comprising six SNPs for eye colour and 13 markers for hair colour have been suggested to reliably predict these traits in European populations. ${ }^{29,30}$

\footnotetext{
${ }^{1}$ Institute of Medical Informatics and Statistics, Christian-Albrechts University Kiel, Kiel, Germany; ${ }^{2}$ Institute of Legal Medicine, Christian-Albrechts University Kiel, Kiel, Germany; ${ }^{3}$ Institute of Clinical Molecular Biology, Christian-Albrechts University Kiel, Kiel, Germany

${ }^{*}$ Correspondence: Dr N von Wurmb-Schwark, Institute of Legal Medicine, Christian-Albrechts University Kiel, University Hospital Schleswig-Holstein, Arnold-Heller Strasse 12, 24105 Kiel, Germany. Tel: +49 431597 3633; Fax: +49 431597 3612. E-mail: Wurmb-Schwark@petgene.net

${ }^{4}$ These authors contributed equally to this work.

${ }^{5}$ Present address: State Criminal Investigation Department, Berlin, Germany.

${ }^{6}$ Present address: ForScix GmbH, Hamburg, Germany.

Received 16 September 2013; revised 1 April 2015; accepted 7 June 2015; published online 19 August 2015
} 
We aimed to develop a more specific prediction model for a realistic target population of forensic case work from Northern Germany. To this end, we investigated the association between eye, hair and skin colour and 12 candidate pigmentation SNPs in six different genes. These SNPs were partly overlapping with the two previously established marker sets mentioned above. Because of the special role of red hair, we followed a new phenotyping strategy, dividing hair colour into two, possibly independent, sub-phenotypes: the red tint component and the light-dark component. The light-dark component was defined by nine evenly graded types of shading.

After model selection, we evaluated the predictive capability of our models and compared our results with that of the other two marker sets. $^{29,30}$ To obtain unbiased estimates of predictive capability, we adopted a two-stage design for our study with 300 individuals in stage 1, the 'modelling sample', and 100 different individuals in stage 2, the 'estimation sample'. Finally, in addition to deriving population-specific prediction models, we analysed the association between phenotypes and tried to define coherent phenotype groups.

\section{MATERIALS AND METHODS}

\section{Study population}

A total of 400 unrelated individuals (197 male, 203 female) from Northern Germany were recruited for our study between 2010 and 2011. The median age was 27 years (interquartile range: $24-33$ years). All individuals were born in Germany and had German parents and grandparents (self-report). All 400 participants were recruited and investigated in the same way. The first 300 participants were included in the 'modelling sample' of stage 1 (used for
SNP selection), the remaining 100 individuals constituted the 'estimation sample' of stage 2 (prediction evaluation). All participants gave written informed consent before the study. Genotype and phenotype data were deidentified for analysis purposes according to the declaration of Helsinki. The project was approved by the Ethics Committee of the Medical Faculty of Christian-Albrechts University Kiel.

\section{Phenotyping}

Pigmentation phenotypes were documented by photographs taken at daylight conditions and from a distance of $30 \mathrm{~cm}$, using a Canon EOS 400D (Canon Deutschland GmbH, Krefeld, Germany) (18-55 mm focal length). For each participant, one photograph was taken of each eye, the scalp hair and the inner arm. Photographs were normalised using the standard functions in Photoshop 4.0 (Adobe Systems Software, San Jose, CA, USA), and consensus phenotype calling was carried out by two raters by discussion. In the rare cases where no agreement was reached a third party was involved.

Eye colour was divided into three categories, namely blue, green and brown (Figure 1a). Individual skin type was classified applying the Fitzpatrick scheme $^{31}$ to the inner arm. Hair colour type was defined in multitiered fashion. To this end, a collection of coloured hair strands obtained from a hairdresser was categorised into nine evenly graded types of shading, ranging from light blond (type I) to black (type IX) (Figure 1b). Strands of red hair or with red tint were omitted from this classification because it was intended to address the light-dark component only. Then, hair colour was divided into two sub-phenotypes, namely the red tint component (yes/no) and the light-dark component (I-IX). For each individual, the presence of red tint was ascertained (by questioning) in head hair, facial hair (beard), axillary hair or pubic hair. If an individual had red head hair, this was noted separately to enable a separate
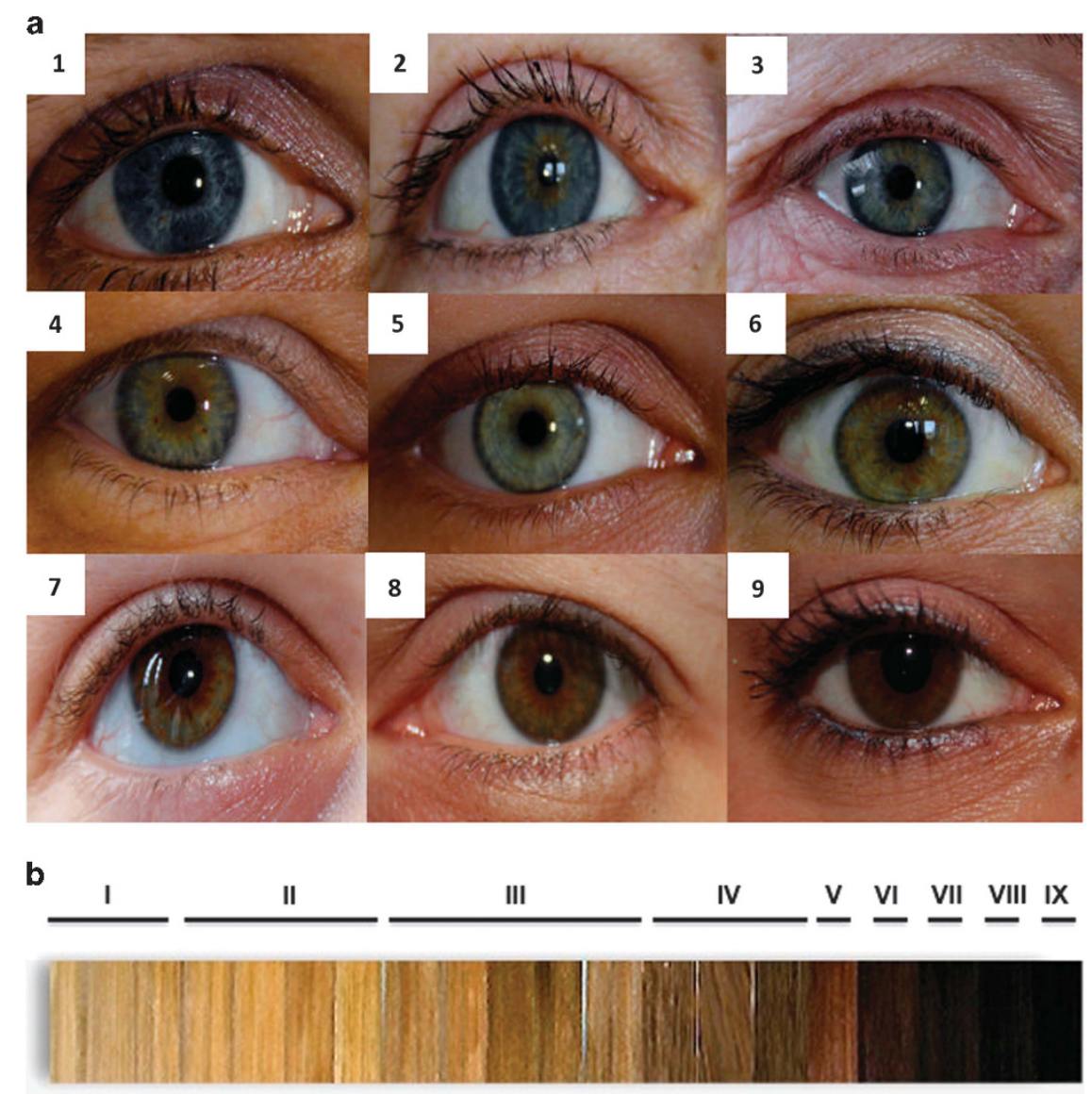

Figure 1 Definition of eye and hair colour phenotypes. (a) Classification of eye colour; blue: 1 - pure blue, 2 - blue-brown, 3 - blue-green; green: 4 - bluegreen-brown, 5 - pure green, 6 - green-brown; brown: 7 - amber, 8 - brown-green, 9 - pure brown. (b) Hair strands used for hair colour categorisation; light blond - I, blond - II, dark blond - III, ash - IV, light brown - V, brown - VI, dark brown - VII, black-brown - VIII, black - IX. 
analysis for this special phenotype. The light-dark component was determined by reference to the hair strand collection mentioned above. Here, individuals with recognisable red tint were classified according to their basic hair colour type. For example, strawberry blonds were deemed class I (blond), whereas people with auburn hair were classified as one of IV, V or VI (brown). Although this was possible for 22 red-haired individuals, four had no definable basic hair colour. These were excluded from the analysis of the light-dark component. No participants with exclusively white hair were included in the study. When a participant had dyed hair, the original hair colour was determined by the hairline.

\section{Genotyping}

Buccal swabs (COPAN) were taken from all 400 participants and DNA was extracted using Chelex 100 (Walsh $e^{2} a^{\beta 2}$ ). In a comprehensive PubMed search, 12 SNPs were identified as promising candidates for further analysis using the following criteria (Table 1, Supplementary Table S1): large odds ratio (OR), validation in several independent studies, large sample sizes and adequate population backgrounds, low to no linkage disequilibrium with other candidate markers and suitability for genotyping in a single assay. In addition to the 12 SNPs, participants were genotyped for rs1426654 (SLC24A5), rs1129038 (HERC2) and rs1667394 (OCA2). SNP rs1426654 is a European ancestry marker (Giardina et $a^{\beta 3}$ ) used to control population background. SNPs rs1129038 and rs1667394 served as genotyping quality markers because they are in perfect LD with candidate SNPs rs12913832 and rs916977 respectively (Mengel-From $e t a l^{34}$; Sturm et $a l^{17}$ ).

Primers were designed and checked for possible dimer and hairpin structures using the DNAstar Lasergene v8.1.2 software (DNASTAR, Madison, WI, USA) and BLAST. PCR fragments had to be shorter than $200 \mathrm{bp}$ in order to meet the standards of reliable forensic or ancient DNA analysis.

For DNA amplification, a Multiplex PCR Master Mix (Qiagen, Hilden, Germany) was used in a total reaction volume of $12.5 \mu \mathrm{l}$, with $0.2-0.5 \mathrm{ng}$ template DNA. PCR was performed with a thermal cycler 2700 (Life Technologies, Carlsbad, CA, USA) under the following conditions: (1) $95^{\circ} \mathrm{C}$ $15 \mathrm{~min}$; (2) 35 cycles of $94^{\circ} \mathrm{C} 30 \mathrm{~s}, 64^{\circ} \mathrm{C}$ (SNPs nos 2-5, 7-8, 10 in Table 1) or $58^{\circ} \mathrm{C}$ (SNPs nos $1,6,9,11$ and 12) $90 \mathrm{~s}, 72^{\circ} \mathrm{C} 1 \mathrm{~min}$; and (3) $60^{\circ} \mathrm{C} 30 \mathrm{~min}$. PCR products were purified using ExoSAP-IT (Affymetrix, Santa Clara, CA, USA) according to the manufacturer's protocol. Single-base extension was carried out in a total reaction volume of $7 \mu \mathrm{l}$, including $0.5 \mu \mathrm{l}$ of cleaned PCR products, using the SNaPshot Multiplex Kit (Life Technologies) on the same PCR cycler as before. The single-base extension cycling conditions were as follows: 25 cycles of $96^{\circ} \mathrm{C} 10 \mathrm{~s} ; 55^{\circ} \mathrm{C} 5 \mathrm{~s}$; and $60^{\circ} \mathrm{C} 30 \mathrm{~s}$. Fragment analysis was performed with the ABI Prism 3130 Genetic Analyzer (Life Technologies) using GeneMapper v3.2 (Life Technologies). For more information on primer sequences and concentrations, see Supplementary Table S2a.

Table 1 Candidate SNPs investigated for an association with different pigmentation traits

\begin{tabular}{|c|c|c|c|c|}
\hline No. & Gene & SNP-ID & Alleles & Reference \\
\hline 1 & HERC2 & rs12913832 & $\mathrm{G} / \mathrm{A}$ & Sturm et al ${ }^{17}$ \\
\hline 2 & HERC2 & rs916977 & $G / A$ & Kayser et a/18 \\
\hline 3 & OCA2 & rs1800407 & $G / A$ & Sturm et al ${ }^{17}$ \\
\hline 4 & OCA2 & rs7495174 & $G / A$ & Duffy et al ${ }^{15}$ \\
\hline 5 & OCA2 & rs4778138 & $\mathrm{G} / \mathrm{A}$ & Duffy et all5 \\
\hline 6 & OCA2 & rs4778241 & $\mathrm{C} / \mathrm{A}$ & Duffy et al ${ }^{15}$ \\
\hline 7 & $M C 1 R$ & rs1805007 & $\mathrm{C} / \mathrm{G} / \mathrm{T}^{\mathrm{a}}$ & Sulem et aR1 \\
\hline 8 & $M C 1 R$ & rs1805008 & $\mathrm{C} / \mathrm{T}$ & Sulem et $a^{R 1}$ \\
\hline 9 & $M C 1 R$ & rs1805009 & $\mathrm{G} / \mathrm{C}$ & Valverde et aRo \\
\hline 10 & IRF4 & rs12203592 & $\mathrm{G} / \mathrm{A}$ & Han et $a R$ \\
\hline 11 & $S L C 24 A 4$ & rs12896399 & $\mathrm{G} / \mathrm{T}$ & Sulem et a ${ }^{21}$ \\
\hline 12 & $T Y R$ & rs1393350 & $G / A$ & Sulem et $a^{R 1}$ \\
\hline
\end{tabular}

Abbreviation: SNP, single-nucleotide polymorphism.

Reference: first report of an association between the respective SNP and a pigmentation trait. ${ }^{a}$ Only alleles $\mathrm{C}$ and $\mathrm{T}$ of SNP rs1805007 (MC1R) were observed in our study.
The model proposed by Walsh $e t a^{30}$ for the prediction of eye colour is based upon six SNPs. Five of these had been genotyped in all our study participants before (Table 1, SNPs nos 1, 3, 10-12). The 100 individuals of stage 2 were additionally genotyped for rs16891982 (SLC45A2) as described above, with an annealing temperature of $58^{\circ} \mathrm{C}$ in the first PCR. For more information on primer sequences and concentrations, see Supplementary Table S2b.

The model devised by Branicki et a ${ }^{29}$ for predicting hair colour is based upon 13 single or compound markers. Three of these were also included in our set of candidate SNPs (Table 1, SNPs nos. 1, 3, 10) and were genotyped in all participants. The 100 stage 2 individuals were also genotyped for the remaining 10 markers, namely two compound markers in MC1R and rs1042602 (TYR), rs4959270 (EXOC2), rs28777 (SLC45A2), rs683 (TYRP1), rs2402130 (SLC24A4), rs12821256 (KITLG), rs16891982 (SLC45A2) and rs2378249 (ASIP). SNPs were analysed as described above, with an annealing temperature of $58^{\circ} \mathrm{C}$ for the first PCR. The MC1R markers were analysed by sequencing the whole locus. To this end, a $1080 \mathrm{bp}$ fragment was amplified and sequenced with an ABI Prism $3130 \times 1$ Genetic Analyzer using the Big Dye Terminator v3.1 Cycle Sequencing Kit (both Life Technologies), following the manufacturer's protocols. See Supplementary Table S2c for more information on primers used in this study.

Genotype and phenotype data of this study were submitted to the European Genome-phenome Archive (EGA, https://ega.crg.eu) with study accession number EGAS00001001174 (sample/proband ids EGAN00001268626EGAN00001269025).

\section{Statistical analysis}

Genotypes for all markers of the two previously published models (or marker sets) (Branicki et $a^{29}$; Walsh et $a l^{30}$ ) were only available for 100 individuals in our study (stage 2). To compare the predictive capability of the two marker sets with a model derived specifically for our target population, the 300 individuals of stage 1 were used to detect significant genotype-phenotype associations and to create an appropriate prediction model. Data from stage 2 then served for estimation and comparison of the predictive capability (sensitivity, specificity, predictive accuracy, area under the receiver operating characteristic curve (AUC)) for each new model and the two previously published models (Branicki et $a 2^{29}$; Walsh et $\left.a{ }^{30}\right)$. For illustration, we also performed model selection and prediction evaluation on the whole data set (ie, stages 1 and 2 combined), using cross-validation to estimate sensitivity and specificity.

Sample size calculations indicated that $\sim 100$ individuals per group would suffice to detect an OR of 3 as nominally significant, depending upon the minor allele frequency of the SNP of interest, and 150 individuals per group after Bonferroni adjustment (12 SNPs tested, $80 \%$ power, 5\% significance level). Stage 1 therefore comprised 300 individuals. The association between a given trait (ie, eye colour, hair colour/red tint, hair colour/light-dark component, skin colour) and a candidate SNP was tested for statistical significance using regression models. To allow for scarce genotypes, we performed permutation tests (100 000 permutations) in addition to standard asymptotic tests. Since $P$-values were not found to be notably different, only $P$-values from permutation tests will be given. Each SNP was analysed both individually (simple regression) and in combination with other candidate SNPs (multiple regression with backward selection), also allowing for possible SNP-SNP interactions. Genotypic, additive allelic, dominant and recessive models were considered for each SNP. Results, however, will be presented for the additive model only because this model required the least parameters but yielded consistently large effects. To derive robust prediction models, phenotypes were categorised in various ways. Dependent on the scaling of the outcome, we performed logistic, linear, ordinal (proportional odds) and/or multinomial logistic regression. Since blue was by far the most frequent eye colour in our study, the analysis of eye colour was confined to the discrimination between blue and non-blue. For hair colour, red tint was treated as a dichotomous outcome whereas the light-dark component was treated in three different ways, either as dichotomous (blond versus non-blond), ordinal or quantitative (nine types of increasing darkness). Skin colour was treated either as dichotomous (types I-II versus types III-IV) or as ordinal. Model selection was performed differently for the four traits. For eye colour and red tint, SNPs that remained significant in the multiple logistic regression analysis after backward selection and adjustment for multiple testing 
were included in the final model. For the light-dark component of hair colour, and for skin colour, a SNP had to be significant in all or in all but one of the multiple regression analyses after backward selection using different outcome definitions (ie, at least two of three analyses for the light-dark component, at least one of two analyses for skin colour).

The relationships between traits were analysed using logistic regression analysis, treating one trait as the dependent variable and the other traits as independent variables, both with and without the additional inclusion of SNP genotypes. All four traits were encoded as dichotomous variables in these analyses. Model selection was again performed by backward selection. Multidimensional scaling was used to detect and visualise patterns in the phenotype data.

The predictive capability of a derived model was evaluated by means of the phenotype probability $\pi$ from logistic regression analysis. This was done only for dichotomous outcomes (eg, blue versus non-blue eye colour). If $\pi>0.5$, the corresponding phenotype was assumed to be present. Predictive capability was quantified by the sensitivity, specificity, predictive accuracy and AUC of the model in question.

All statistical analyses were performed with R v2.10.1 (R Development Core $\mathrm{Team}^{35}$ ) unless indicated otherwise. Hardy-Weinberg equilibrium was assessed by means of the exact test implemented in R package genetics (Warnes et a ${ }^{\beta 6}$ ). Package MASS was used for ordinal and multinomial regression (Venables and Ripley $^{37}$ ). Permutation tests of the linear and logistic regression models were performed with package glmperm (Werft et $a^{38}$ ). For ordinal regression models, permutation tests were programmed in house. The predictive capabilities of different models were evaluated with packages DiagnosisMed $\left(\right.$ Brasi $\left.^{39}\right)$ and pROC (Robin $e t \mathrm{al}^{40}$ ). The proportion of phenotype heritability explained by a given marker was calculated according to So et al. ${ }^{41}$ Note that these estimates apply to single markers and do not take into account the characteristics of the respective regression models. Furthermore, these estimates refer to the liability scale and therefore tend to be higher than on the observation scale.

Sample size calculations were performed with the GPower software v3.0.8. ${ }^{42}$ All tests were two-sided and a $P$-value smaller than 0.05 was considered nominally statistically significant. $P$-values were adjusted for multiple testing using the Bonferroni method.

\section{RESULTS}

\section{Hardy-Weinberg equilibrium}

After adjustment for multiple testing, none of the SNPs showed a significant deviation from the Hardy-Weinberg equilibrium.

\section{Eye colour}

Since blue was by far the most frequent eye colour in our study population, we confined our analysis of eye colour to the discrimination between blue and non-blue (Supplementary Table S3a; for the stratified genotype distribution, see Supplementary Table S4; for the eye colour categorization, see Figure 1a). When analysed individually, five SNPs were found to be significantly associated with blue eye colour (Supplementary Table S3a), namely rs12913832, rs916977 (both HERC2), rs7495174, rs4778241 and rs4778138 (all OCA2). When all 12 candidate SNPs were included in a multiple logistic regression analysis, backward selection left only SNPs rs12913832 (HERC2) and rs1800407 (OCA2) with a significant phenotype association after adjustment for multiple testing (Table 2). Of these two SNPs, rs12913832 showed by far the strongest effect $\left(P<1.0 \times 10^{-5}, \quad p_{\text {adj }}<1.2 \times 10^{-4}, \quad O R=40.0, \quad 95 \% \mathrm{CI}=18.3-87.5\right)$, although the effect of rs1800407 was still of considerable size $\left(P=0.0014, p_{\text {adj }}=0.017, \quad \mathrm{OR}=4.9,95 \% \mathrm{CI}=1.8-13.6\right)$. When the expected prevalence of blue eye colour was calculated for each rs12913832/rs1800407 genotype combination (Supplementary Table $\mathrm{S} 3 \mathrm{~b})$, reasonable agreement with the observed frequencies was observed. Models of brown versus non-brown eye colour and blue versus brown eye colour revealed similarly strong genotype-phenotype associations. Moreover, the results were largely independent of whether dichotomous, ordinal or multinomial regression analyses were performed. When modelling brown versus non-brown, blue versus brown and in the ordinal regression analysis of eye colour, SNP rs4778138 (OCA2) showed a nominally significant association with the respective trait in a multiple regression that did not, however, withstand correction for multiple testing.

\section{Hair colour - red tint}

Hair colour was defined by two distinct traits, namely a light-dark component and whether red tint was visible in the scalp or body hair, or not. Only two SNPs in the MC1R gene were found to be significantly associated with the red tint trait in a multiple regression analysis (Supplementary Table S5a; for the corresponding genotype data, see Supplementary Table S6). Of these, rs1805007 showed the stronger effect (Table 2; $P<1.0 \times 10^{-5}, p_{\text {adj }}<1.2 \times 10^{-4}, \mathrm{OR}=5.4$, $95 \% \mathrm{CI}=2.8-10.3)$ whereas only a moderate association was noted for $\operatorname{rs1} 1805008\left(P=1.0 \times 10^{-4}, p_{\text {adj }}=0.0012, \mathrm{OR}=3.5,95 \% \mathrm{CI}=1.9-6.6\right)$. As with eye colour, the observed and expected genotype-specific prevalence of red tint were found to agree well (Supplementary Table S5b). Similar results were obtained when red hair colour (26 individuals) was analysed instead of red tint (102 individuals).

\section{Hair colour - light-dark component}

The light-dark component of hair colour was initially categorised as blond, brown or black, with blond being the predominant type. In addition, we defined nine evenly graded hair colour types of different

Table 2 Model-based genotype-phenotype association for four pigmentation traits for stage 1

\begin{tabular}{|c|c|c|c|c|}
\hline Trait & SNP (gene) & $p\left(p_{a d j}\right)$ & OR $(95 \% C l)$ & Explained heritability ${ }^{a}$ \\
\hline \multirow[t]{2}{*}{ Eye colour (blue) dichotomous } & rs12913832 (HERC2) & $<1.0 \times 10^{-5}\left(1.2 \times 10^{-4}\right)$ & $40.0(18.3-87.5)$ & $46 \%$ \\
\hline & rs1800407 (OCA2) & $0.0014(0.017)$ & $4.9(1.8-13.6)$ & $1 \%$ \\
\hline \multirow[t]{2}{*}{ Hair colour (red tint) dichotomous } & rs1805007 (MC1R) & $<1.0 \times 10^{-5}\left(1.2 \times 10^{-4}\right)$ & $5.4(2.8-10.3)$ & $14 \%$ \\
\hline & rs1805008 (MC1R) & $1.0 \times 10^{-4}(0.0012)$ & $3.5(1.9-6.6)$ & $7 \%$ \\
\hline \multirow[t]{2}{*}{ Hair colour (light-dark, I-IX)b ordinal } & rs12913832 (HERC2) & $<1.0 \times 10^{-5}\left(1.2 \times 10^{-4}\right)$ & $2.9(1.9-4.4)^{c}$ & $5 \%$ \\
\hline & rs12203592 (IRF4) & $<1.0 \times 10^{-5}\left(1.2 \times 10^{-4}\right)$ & $3.6(2.0-6.3)^{c}$ & $5 \%$ \\
\hline \multirow[t]{3}{*}{ Skin type (I-IV) ordinal } & rs1805008 (MC1R) & $6.0 \times 10^{-5}\left(7.2 \times 10^{-4}\right)$ & $3.0(1.8-5.1)^{\mathrm{c}}$ & $8 \%$ \\
\hline & rs1805007 (MC1R) & $0.0035(0.042)$ & $2.5(1.4-4.3)^{c}$ & $5 \%$ \\
\hline & rs4778138 (OCA2) & $4.9 \times 10^{-4}(0.0059)$ & $3.2(1.6-6.2)^{c}$ & $4 \%$ \\
\hline
\end{tabular}

Abbreviations: $\mathrm{Cl}$, confidence intervals; OR, odds ratio; SNP, single-nucleotide polymorphism.

$p_{\text {adj: }}$ : Bonferroni-adjusted $P$-value (adjusted for 12 tests).

The proportion of the trait heritability explained by a given variant on the liability scale was assessed as described by So et a ${ }^{A 1}$ (without consideration of other markers interacting or in LD).

bIn the analysis of the light-dark component of hair colour, two individuals with pure red hair colour were removed.

cORs for ordinal phenotypes correspond to a proportional odds model. 
shading, ranging from light blond (type I) to black (type IX) (Figure 1b). The most consistent association with hair colour was noted for rs12913832 (HERC2), which showed a statistically significant effect in all three analyses (dichotomous blond versus non-blond, ordinal and linear regression of the light-dark component; Supplementary Table S8a). SNP rs12203592 (IRF4) also showed a highly significant association with the light-dark component of hair colour, but was less strongly associated with the blond versus non-blond trait. The two SNPs were also the only ones included in the final model of the genotype-phenotype relationship (Table 2; rs12913832: $\quad P<1.0 \times 10^{-5}, \quad p_{\text {adj }}<1.2 \times 10^{-4}, \quad$ OR $=2.9, \quad 95 \%$ $\mathrm{CI}=1.9-4.4 ; \mathrm{rs} 12203592: P<1.0 \times 10^{-5}, p_{\mathrm{adj}}<1.2 \times 10^{-4}, \mathrm{OR}=3.6$, $95 \% \mathrm{CI}=2.0-6.3$ ). For further details on the genotype-phenotype relationship of the light-dark component, see Supplementary Tables S7 and S8.

\section{Skin colour}

Skin colour was categorised into four types on using the Fitzpatrick scale. ${ }^{31}$ Our genetic association analysis was performed twice, once discriminating between skin types I-II and III-IV, and once by ordinal regression of the four skin types (for details see Supplementary Tables S9 and S10). SNPs rs1805007, rs1805008 (both MC1R) and rs4778138 (OCA2) were selected for the final model of the genotype-phenotype relationship and showed a consistent albeit moderate association with skin type (Table 2; rs1805008: $P=6.0 \times 10^{-5}, p_{\text {adj }}=7.2 \times 10^{-4}, \mathrm{OR}=3.0$, 95\% CI $=1.8-5.1 ;$ rs 1805007: $P=0.0035, p_{\text {adj }}=0.042, \mathrm{OR}=2.5,95 \%$ $\mathrm{CI}=1.4-4.3 ; \mathrm{rs} 4778138: P=4.9 \times 10^{-4}, p_{\mathrm{adj}}=0.0059, \mathrm{OR}=3.2,95 \%$ $\mathrm{CI}=1.6-6.2)$. For further details on the genotype-phenotype relationship of skin type, see Supplementary Tables S9 and S10.

\section{Association between phenotypes}

As was to be expected, the four pigmentation traits were not statistically independent. Thus, the phenotypes blue eye colour and blond hair colour and the phenotypes red tint and fair skin were strongly associated with one another even when the respective genotypes from the final genotype-phenotype models (Table 2) were taken into account (Table 3). Interestingly, red tint and blond hair colour were not significantly associated with one another in our data.
Joint multidimensional scaling analysis of the four traits in both stages combined resulted in four distinct clusters (Figure 2). These clusters were determined completely by blue eye colour and red tint which therefore seem to be the most differentiating pigmentation traits. The same clusters were also found in stages 1 and 2 individually (data not shown). For information on the exact phenotypic composition of the four clusters, see Supplementary Table S11.

\section{Predictive capability of SNP-based models}

We next assessed the predictive capability of the different SNP- and phenotype-based models derived in our study (Table 4a). In the process, we used the most prevalent phenotype as the reference category for eye and hair colour (light-dark component), that is, blue eyes and blond hair, to ensure sufficient sample size. SNP-based prediction was found to perform best for eye colour, with a sensitivity of $93 \%$, a reasonable specificity of $59 \%$, a predictive accuracy of $84 \%$ and an AUC of $77 \%$. Additional inclusion of blond hair colour as a predictor of eye colour increased the predictive capability only marginally. Prediction of red tint also yielded comparatively high accuracy (74-77\%) owing to the high specificity of the SNP genotypes (97-99\%) and the low prevalence of the trait (31\%), but had low sensitivity (19-32\%). The AUC was approximately 75\%. Blond hair was predicted moderately well by SNP genotypes (83\% sensitivity, $67 \%$ specificity, $76 \%$ accuracy, $76 \%$ AUC). Interestingly, when phenotypes were included in the logistic regression model, backward selection excluded SNP rs12913832 (HERC2) to the benefit of blue eye colour. However, the ensuing model gave poorer predictive power (67\% accuracy, $71 \%$ AUC) owing to a reduced sensitivity (67\%). Finally, fair skin (types I and II) could be predicted with very high specificity (100\%) using three SNPs, but sensitivity was low (9\%), thereby resulting in a predictive accuracy of only $51 \%$ and an AUC of $64 \%$. Only a slight improvement was achieved by the inclusion of other pigmentation traits as predictors.

\section{Comparison with previously proposed marker sets}

We compared our eye colour model comprising only rs12913832 (HERC2) and rs1800407 (OCA2) (Table 2) to the six SNPs of the socalled 'IrisPlex', proposed by Walsh et al. ${ }^{30}$ Because of the high

Table 3 Associations between dichotomous pigmentation traits for stage 1

\begin{tabular}{|c|c|c|c|c|}
\hline Pigmentation trait & $p\left(p_{a d j}\right)$ & $p\left(p_{a d j}\right)^{a}$ & OR $(95 \% \mathrm{Cl})$ & OR $(95 \% C I)^{\mathrm{a}}$ \\
\hline \multicolumn{5}{|l|}{ Eye colour (blue) } \\
\hline Hair colour (blond) & $<1.0 \times 10^{-5}\left(3.0 \times 10^{-5}\right)$ & $0.0047(0.014)$ & $3.6(2.1-6.2)$ & $2.7(1.3-5.5)$ \\
\hline \multicolumn{5}{|l|}{ Hair colour (red tint) } \\
\hline Skin type (fair, I-II) & $<1.0 \times 10^{-5}\left(3.0 \times 10^{-5}\right)$ & $3.0 \times 10^{-4}\left(9.0 \times 10^{-4}\right)$ & $4.1(2.4-7.2)$ & $3.1(1.7-5.6)$ \\
\hline \multicolumn{5}{|l|}{ Hair colour (blond) } \\
\hline Eye colour (blue) & $1.0 \times 10^{-5}\left(3.0 \times 10^{-5}\right)$ & $<1.0 \times 10^{-5}\left(3.0 \times 10^{-5}\right)^{c}$ & $3.6(2.1-6.3)$ & $4.4(2.5-7.8)^{\mathrm{c}}$ \\
\hline \multicolumn{5}{|l|}{ Skin type (fair, I-II) } \\
\hline Hair colour (red) & $<1.0 \times 10^{-5}\left(3.0 \times 10^{-5}\right)$ & $<1.0 \times 10^{-5}\left(3.0 \times 10^{-5}\right)^{d}$ & $5.7(3.1-10.2)$ & $5.7(3.1-10.2)^{d}$ \\
\hline Hair colour (blond) & $2.7 \times 10^{-4}\left(8.1 \times 10^{-4}\right)$ & $2.7 \times 10^{-4}\left(8.1 \times 10^{-4}\right)^{d}$ & $2.9(1.6-5.2)$ & $2.9(1.6-5.2)^{d}$ \\
\hline
\end{tabular}

Abbreviations: $\mathrm{Cl}$, confidence interval; OR, odds ratio; SNP, single-nucleotide polymorphism.

$p_{\text {adj: }}$ Bonferroni-adjusted $P$-value (three tests). All associations were evaluated in a multiple logistic regression analysis treating the remaining three traits as influential variables, and by backward selection.

a $P$-values and ORs are from a multiple logistic regression analysis including the predictive SNPs from Table 2 as influential variables.

bIn the analysis of the light-dark component of hair colour, two individuals with pure red hair colour were removed.

CMultiple logistic regression analysis including the predictive SNPs of Table 2 eventually disregarded rs12913832 (HERC2) after backward selection.

dMultiple logistic regression analysis eventually disregarded all predictive SNPs from Table 2 after backward selection. 


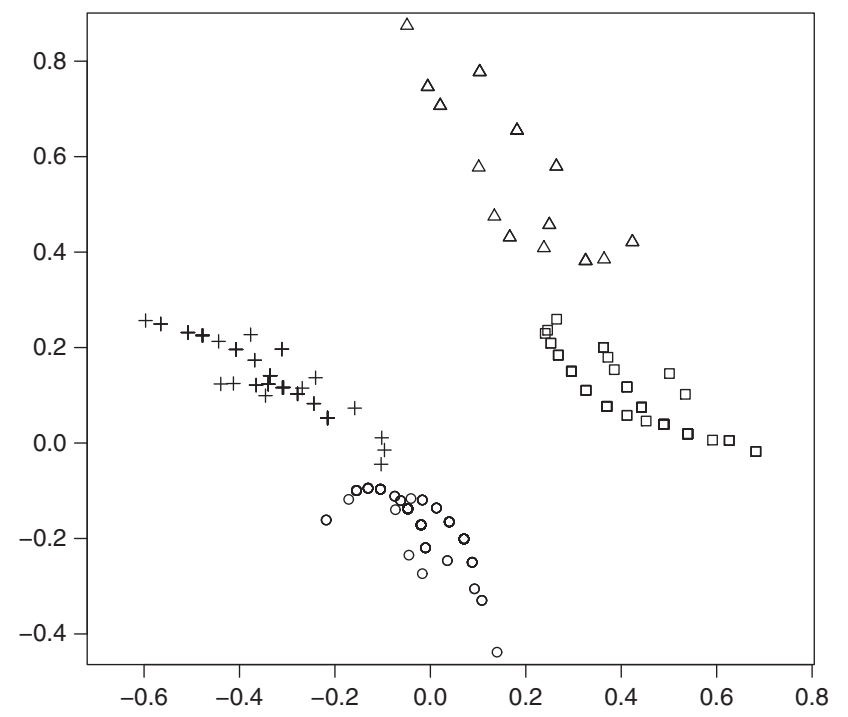

Figure 2 Multidimensional scaling analysis of four pigmentation traits. Squares: blue eye colour, red tint; circles: blue eye colour, no red tint; triangles: no blue eye colour, red tint; crosses: no blue eye colour, no red tint. All individuals of stages 1 and 2 were included in the analysis except four individuals with pure red hair. Phenotypes were coded as follows: eye colour, ordinal (blue, green, brown); hair colour - red tint, dichotomous; hair colour - light-dark component, ordinal (I to IX); skin colour, ordinal (I to IV).

prevalence of blue eye colour and the low prevalence of green and brown eye colour in our study population, we focused upon the discrimination between blue and non-blue eye colour. For comparison, we also considered a model based upon major SNP rs12913832 (HERC2) alone. All three models yielded comparable predictive accuracy but the AUC of the IrisPlex model was found to be considerably larger ( $89 \%$ versus $77 \%$, Table 4 b). For hair colour, we compared our models (Table 2) to the 13 single or compound markers proposed by Branicki et al. ${ }^{29}$ The latter performed worse than our model for red tint. For blond hair, its predictive capacity was rather low, with an accuracy of $56 \%$ and an AUC of $57 \%$, and was even outperformed by a model including only the major SNP (Table $4 \mathrm{~b}$ ). Interestingly, the predictive accuracy for blond hair and red tint was similar for the selected models of this study and a model incorporating only the respective major SNP, ie, either rs 12913832 (HERC2) or rs1805007 (MC1R).

\section{Analysis of the whole data set}

We also analysed our whole data set (ie, stages 1 and 2 combined) and the results were consistently found to be similar to those of the twotiered analysis (Supplementary Tables S12-S17). Owing to the larger sample size occasionally more significant results emerged. Thus, two additional markers (rs1805008 in MC1R and rs12896399 in SLC24A4) were significantly associated with the light-dark component of hair colour, and one additional marker (rs7495174 in OCA2) emerged for skin colour. For red tint, the same two markers in $M C 1 R$ as before were found to be significant. For eye colour the marker rs1800407 in OCA2 was now disregarded in the final model, therefore this now only consists of the main marker rs12913832 in HERC2.

When the associations between phenotypes were investigated, a new significant relationship between blond hair colour and fair skin emerged. Still, no significant association between red tint and blond hair colour was found.
For blue eye colour, the predictive capability of the main marker alone as estimated by cross-validation in the whole data set was higher than the capability estimated from stage 2 for a model of one or two markers selected from stage 1. Similar results were obtained for skin colour. For red tint and blond hair colour, by contrast, the stage 2-based estimates for models derived in stage 1 were found to be the higher ones.

\section{DISCUSSION}

In our study population, only six of 12 candidate SNPs investigated in stage 1 were significantly associated with a pigmentation phenotype of eye, hair or skin. These SNPs were located in four genes, namely HERC2, MC1R, IRF4 and OCA2. The remaining SNPs showed no consistent association in the different analyses performed. Due to the limited sample size (300 individuals in stage 1), however, we cannot exclude that weak effects may have been overlooked. This possibility is also highlighted by the fact that an analysis of the whole data set yielded more significantly associated markers than the two-tiered approach. Larger sample sizes certainly would have revealed more genes to be associated with the (polygenic) pigmentation traits of interest.

The marker set previously proposed by Walsh et al, ${ }^{30}$ including six SNPs, achieved the best predictive capability for blue eye colour. This implies that it would also be well suited for use in the comparatively homogeneous Northern German population under study here. Notably, for the red tint component of hair colour, the model including only major SNP rs1805007 in the MC1R gene performed slightly better in our study than the model suggested by Branicki et $a,^{29}$ which includes 13 single or compound markers. However, it must be taken into account that Branicki et a ${ }^{29}$ considered only red hair, and not red tint, as was done in our study. Using the same 13 markers to predict blond hair colour, their predictive capability turned out to be even poorer. We observed that the Branicki model was much inferior to major SNP rs12913832 (HERC2) alone and performed only slightly better than mere chance prediction in the population under study. Thus, the comprehensive prediction model previously suggested for hair colour did not achieve convincing predictive results in our population whilst a model comprising fewer (or only a single) marker(s) performs equally well or even better.

The main goal of our analysis was to develop a prediction model with a view to its practical application in a realistic target population, and to compare its predictive capability to that of previously proposed marker sets. We are fully aware that some of our results may only apply to Northern Germany. Even though population genetic variation is known to be small in Europe ${ }^{43-45}$ more markers or different markers may be required for accurate phenotype prediction in other or less homogeneous populations. Moreover, the power of forensic DNA phenotyping depends upon the prevalence of the pigmentation phenotype(s) in question, and these frequencies differ considerably between European countries (as is clearly demonstrated in the case of red hair). Instead of sensitivity and specificity, the negative and positive predictive values of a given model are more important parameters for practical use, and these are a function of the prevalence. Nevertheless, at least for blue eye colour, our evaluation of the markers proposed by Walsh et $a l^{30}$ gave similar results as a Europe-wide evaluation, by the same group, ${ }^{46}$ of a similar prediction model proposed by Liu et al. ${ }^{47}$ However, since Northern Germany constitutes only a very small segment of the European gene pool, the general validity of our conclusion needs to be clarified in future studies. For worldwide samples, different models may be required and the addition of ancestral markers may be worthwhile in these instances, owing to the preponderance of brown eye colour and dark 
Table 4a Predictive capability of several prediction models for pigmentation phenotypes (stage 2 only). Capability of selected models to predict dichotomous pigmentation phenotypes

\begin{tabular}{|c|c|c|c|c|c|c|}
\hline Phenotype & Prevalence & Predictors & Sensitivity ${ }^{a}$ & Specificity $^{a}$ & Accuracy ${ }^{\mathrm{a}}$ & $A \cup C^{a}$ \\
\hline \multirow[t]{4}{*}{ Eye colour (blue) } & 73 & rs12913832 (HERC2) & $93(85-97)$ & $59(41-75)$ & $84(76-90)$ & $77(67-88)$ \\
\hline & & rs1800407 (OCA2) & & & & \\
\hline & & rs1800407 (OCA2) & & & & \\
\hline & & hair colour (blond) & & & & \\
\hline \multirow[t]{3}{*}{ Hair colour (red tint) } & 31 & rs1805007 (MC1R) & $19(9-36)$ & $99(92-100)$ & $74(65-82)$ & $75(65-85)$ \\
\hline & & rs1805008 (MC1R) & & & & \\
\hline & & skin type (fair, I-II) & & & & \\
\hline \multirow[t]{4}{*}{ Hair colour (blond) } & 53 & rs12913832 (HERC2) & $83(70-91)$ & 67 (53-79) & $76(66-83)$ & $76(67-85)$ \\
\hline & & rs12203592 (IRF4) & & & & \\
\hline & & rs12203592 (IRF4) & $67(54-78)$ & $67(53-79)$ & $67(58-76)$ & $71(62-80)$ \\
\hline & & eye colour (blue) & & & & \\
\hline Skin type (fair, I and II) & & hair colour (blond) & & & & \\
\hline
\end{tabular}

Abbreviation: AUC: area under the receiver operating characteristic curve.

The predictive capabilities were estimated from stage 2 individuals only $(n=100)$. Two individuals with pure red hair were excluded from the analysis of blond hair colour. All numerical figures are percentages.

aFigures in brackets denote the respective $95 \%$ confidence interval.

Table 4b Predictive capability of several prediction models for pigmentation phenotypes (stage 2 only). Comparative analysis of prediction models for pigmentation phenotypes (present study, Walsh et al, ${ }^{30}$ Branicki et $a^{29}$ )

\begin{tabular}{|c|c|c|c|c|}
\hline Model & Sensitivity ${ }^{\mathrm{a}}$ & Specificity ${ }^{a}$ & Accuracy $^{\mathrm{a}}$ & $A \cup C^{a}$ \\
\hline \multicolumn{5}{|l|}{ Eye colour (blue) } \\
\hline Walsh et $a^{\beta 0}$ & $90(82-95)$ & $63(44-78)$ & 83 (74-89) & $89(81-96)$ \\
\hline rs12913832 (HERC2) & 90 (82-95) & $63(44-78)$ & 83 (74-89) & $77(67-87)$ \\
\hline \multicolumn{5}{|l|}{ Hair colour (blond) } \\
\hline rs12913832 (HERC2) & 88 (77-95) & $57(42-70)$ & $73(64-81)$ & $73(64-81)$ \\
\hline \multicolumn{5}{|l|}{ Hair colour (red tint) } \\
\hline Branicki et $a{ }^{29}$ & $26(14-43)$ & $88(78-94)$ & $69(59-77)$ & $62(50-75)$ \\
\hline Present study & $19(9-36)$ & $99(92-100)$ & $74(65-82)$ & $75(65-85)$ \\
\hline rs1805007 (MC1R) & $29(16-47)$ & $96(88-99)$ & $75(66-82)$ & $62(54-71)$ \\
\hline
\end{tabular}

Abbreviation: AUC, area under the receiver operating characteristic curve.

Predictive capabilities were estimated from stage 2 individuals only $(n=100)$. Two individuals with pure red hair were excluded from the analysis of blond hair colour. All numerical figures are Predictive capabilities were estimated from stage 2 individuals only

aFigures in brackets denote the respective $95 \%$ confidence interval.

skin or hair colour in some regions. Such markers were successfully applied before. In the respective studies ${ }^{28,48}$ SNP rs12913832 (HERC2) again had the largest impact on eye colour prediction.

One reason for the poor performance of the blond hair markers proposed by Branicki et al ${ }^{29}$ may be that the original study used a rather small sample of 385 individuals to estimate a large number of parameters (13 influential variables and four response hair categories) in a multinomial model, which rendered the analysis prone to overfitting. The use of non-validated SNPs with minor or no effect and the possibility of population-specific genotype-phenotype relationships imply that the development of ever refined models with large numbers of SNPs may result in prediction tools that are no longer robust. Consequently, weak-to-moderate effects could often not be replicated. ${ }^{11,18,49}$ Recently, the model originally proposed by Branicki et $a 2^{29}$ has been refined on the basis of a large European data set. The resulting so-called 'HIrisPlex' ${ }^{50}$ comprises all previously proposed 13 markers plus 8 additional markers also considered by Branicki et al. ${ }^{29}$ Because of its recency we have not been able to determine whether the HIrisPlex produces better results than the smaller model in the Northern German population.

For simplicity and comparability, phenotype prediction employed a probability threshold of 0.5 in our analyses. Possibly, other thresholds 
might produce better results for some of the models. Another aspect is that individuals with a phenotype probability around 0.5 are difficult to classify correctly anyway. One possibility to overcome this problem would be to apply a high and a low threshold in the first place, and to treat individuals between these thresholds as 'undetermined'. ${ }^{29,46}$ In this case, specificity and sensitivity of the prediction model could be increased but would result in a large proportion of cases where no prediction is possible. A second option would be to discard thresholds altogether and for the scientist or forensic expert to merely communicate the actual phenotype probability.

Several methods are available for statistical model selection with the aim of prediction. We used regression models with backward selection based upon a likelihood ratio test of the association between influential variables (ie, candidate SNPs) and an outcome of interest. Another popular method is maximisation of the AUC as employed in the studies by Walsh et $a b^{30}$ and Branicki et al. ${ }^{29}$ Here, the individuals of the two different outcome groups are ordered by the predictive probability to belong to group 1, and the AUC measures how well the individuals can be differentiated by individuals of group 1 having higher probabilities than individuals of group 2 (as would be desirable). Maximisation of the AUC is perhaps the most widely used but its applicability has come under critical debate lately. ${ }^{51,52}$ In addition, for small marker sets, estimation of the AUC is imprecise because the corresponding receiver operating curve has only few supporting points. For our study including 12 markers in the full model, but only two to three markers in the final models, we consequently chose a regression approach. Note that the marker sets of Walsh et $a l^{\beta 0}$ and Branicki et a ${ }^{29}$ included a higher number of markers, therefore the estimation of the AUC is easier in those studies.

We noted that a more refined definition of the pigmentation phenotypes yielded more significant genetic associations. This was most pronounced for hair colour which was analysed as a dichotomous (blond versus non-blond), ordinal and quantitative trait (types I-IX). Thus, fine phenotyping may facilitate the detection of more moderate genotype-phenotype associations in the future. Fine phenotyping has been applied with favourable results to eye colour before by Liu et al. ${ }^{24}$ In the same vein, Candille et al ${ }^{11}$ successfully measured pigmentation of eye, skin and hair colour on a quantitative scale.

Our study also highlights that pigmentation, even of one and the same part of the body, is genetically complex. This is best illustrated by hair colour which was decomposed into two different sub-phenotypes lacking significant association with one another in our data. Furthermore, whereas the light-dark component was associated with SNP rs12913832 in the HERC2 gene (the SNP that had a very strong effect on eye colour), red tint was associated with SNP rs1805007 in the $M C 1 R$ gene. Variation in $M C 1 R$, in turn, was also shown to be associated with skin colour.

Of all pigmentation traits investigated in our study, eye colour turned out to be best predictable by SNP genotypes. Even for eye colour, however, these predictions were far from being very reliable. Moreover, as was illustrated by the comparison between our models and those of Walsh et $a l^{30}$ and Branicki et $a l,{ }^{29}$ the incorporation of additional SNPs is likely to achieve only small improvements, if any, in terms of the predictive power in a specific population. For skin colour, no reliable gene-based prediction model could be developed at all. These findings, together with the supposedly highly polygenic nature of the pigmentation traits, suggest that it may simply not be possible in a specific population to predict some of the pigmentation phenotypes with sufficient certainty from a handful of SNP genotypes. Instead, reliable prediction may require the use of a high number of SNPs, for example, as formatted on available DNA microarrays. Not least, similar approaches have been used successfully to facilitate the inference of population affiliation in Europe. ${ }^{43,44,53}$ Finally, our study also revealed that the prediction of one pigmentation phenotype may benefit from using information on other pigmentation phenotypes, if and when such information is available. In the future our method might also be combined with other SNP-based assays, eg, for the determination of human origin.

\section{CONFLICT OF INTEREST}

The authors declare no conflict of interest.

\section{ACKNOWLEDGEMENTS}

We thank all participants in our study. We thank Thomas Scholz for technical assistance and Mrs Tina Hagen-Hurley for revising the English text. Melanie Harder received additional support from the Graduate School 'Human Development in Landscapes' at the Christian-Albrechts University of Kiel.

1 Armstrong BK, Kricker A: The epidemiology of UV induced skin cancer. J Photochem Photobiol B 2001; 63: 8-18.

2 Han J, Colditz GA, Hunter DJ: Risk factors for skin cancers: a nested case-control study within the Nurses' Health Study. Int J Epidemiol 2006; 35: 1514-1521.

3 Cullington HE: Light eye colour linked to deafness after meningitis. BMJ 2001; 322 : 587.

4 Rasmussen M, Li Y, Lindgreen S et al: Ancient human genome sequence of an extinct Palaeo-Eskimo. Nature 2010; 463: 757-762.

5 Bouakaze C, Keyser C, Crubezy E, Montagnon D, Ludes B: Pigment phenotype and biogeographical ancestry from ancient skeletal remains: inferences from multiplexed autosomal SNP analysis. Int J Legal Med 2009; 123: 315-325.

6 Draus-Barini J, Walsh S, Pospiech E et al: Bona fide colour: DNA prediction of human eye and hair colour from ancient and contemporary skeletal remains. Investig Genet 2013; 4: 3

7 Kayser M, Schneider PM: DNA-based prediction of human externally visible characteristics in forensics: motivations, scientific challenges, and ethical considerations. Forensic Sci Int Genet 2009; 3: 154-161.

8 Walsh S, Lindenbergh A, Zuniga SB et al: Developmental validation of the IrisPlex system: determination of blue and brown iris colour for forensic intelligence. Forensic Sci Int Genet 2011; 5: 464-471.

9 Sturm RA: Molecular genetics of human pigmentation diversity. Hum Mol Genet 2009; 18: R9-17.

10 Branicki W, Brudnik U, Wojas-Pelc A: Interactions between HERC2, OCA2 and MC1R may influence human pigmentation phenotype. Ann Hum Genet 2009; 73: 160-170.

11 Candille SI, Absher DM, Beleza S et al: Genome-wide association studies of quantitatively measured skin, hair, and eye pigmentation in four European populations. PLoS One 2012; 7: e48294.

12 Rees JL: Genetics of hair and skin color. Annu Rev Genet 2003; 37: 67-90.

13 Sturm RA, Frudakis TN: Eye colour: portals into pigmentation genes and ancestry. Trends Genet 2004; 20: 327-332.

14 Clark P, Stark AE, Walsh RJ, Jardine R, Martin NG: A twin study of skin reflectance. Ann Hum Biol 1981; 8: 529-541.

15 Duffy DL, Montgomery GW, Chen W et al: A three-single-nucleotide polymorphism haplotype in intron 1 of OCA2 explains most human eye-color variation. Am J Hum Genet 2007; 80: 241-252.

16 Eiberg $\mathrm{H}$, Troelsen J, Nielsen $\mathrm{M}$ et al: Blue eye color in humans may be caused by a perfectly associated founder mutation in a regulatory element located within the HERC2 gene inhibiting OCA2 expression. Hum Genet 2008; 123: 177-187.

17 Sturm RA, Duffy DL, Zhao ZZ et al: A single SNP in an evolutionary conserved region within intron 86 of the HERC2 gene determines human blue-brown eye color. Am J Hum Genet 2008; 82: 424-431.

18 Kayser M, Liu F, Janssens AC et al: Three genome-wide association studies and a linkage analysis identify HERC2 as a human iris color gene. Am J Hum Genet 2008; 82: 411-423.

19 Visser M, Kayser M, Palstra RJ: HERC2 rs12913832 modulates human pigmentation by attenuating chromatin-loop formation between a long-range enhancer and the OCA2 promoter. Genome Res 2012; 22: 446-455.

20 Valverde P, Healy E, Jackson I, Rees JL, Thody AJ: Variants of the melanocytestimulating hormone receptor gene are associated with red hair and fair skin in humans. Nat Genet 1995; 11: 328-330.

21 Sulem P, Gudbjartsson DF, Stacey SN et al: Genetic determinants of hair, eye and skin pigmentation in Europeans. Nat Genet 2007; 39: 1443-1452.

22 Han J, Kraft P, Nan $\mathrm{H}$ et al: A genome-wide association study identifies novel alleles associated with hair color and skin pigmentation. PLoS Genet 2008; 4: e1000074. 
23 Sulem P, Gudbjartsson DF, Stacey SN et al: Two newly identified genetic determinants of pigmentation in Europeans. Nat Genet 2008; 40: 835-837.

24 Liu F, Wollstein A, Hysi PG et al: Digital quantification of human eye color highlights genetic association of three new loci. PLoS Genet 2010; 6: e1000934.

25 Soejima M, Koda Y: Population differences of two coding SNPs in pigmentation-related genes SLC24A5 and SLC45A2. Int J Legal Med 2007; 121: 36-39.

26 Kayser M, de Knijff $P$ : Improving human forensics through advances in genetics, genomics and molecular biology. Nat Rev Genet 2011; 12: 179-192.

27 Tully G: Genotype versus phenotype: human pigmentation. Forensic Sci Int Genet 2007; 1: 105-110.

28 Spichenok O, Budimlija ZM, Mitchell AA et al: Prediction of eye and skin color in diverse populations using seven SNPs. Forensic Sci Int Genet 2011; 5: 472-478.

29 Branicki W, Liu F, van Duijn $\mathrm{K}$ et al: Model-based prediction of human hair color using DNA variants. Hum Genet 2011; 129: 443-454.

30 Walsh S, Liu F, Ballantyne KN, van Oven M, Lao O, Kayser M: IrisPlex: a sensitive DNA tool for accurate prediction of blue and brown eye colour in the absence of ancestry information. Forensic Sci Int Genet 2011; 5: 170-180.

31 Fitzpatrick TB: The validity and practicality of sun-reactive skin types I through VI. Arch Dermatol 1988; 124: 869-871.

32 Walsh PS, Metzger DA, Higuchi R: Chelex 100 as a medium for simple extraction of DNA for PCR-based typing from forensic material. Biotechniques 1991; 10: 506-513.

33 Giardina E, Pietrangeli I, Martinez-Labarga C et al: Haplotypes in SLC24A5 gene as ancestry informative markers in different populations. Curr Genomics 2008; 9 : $110-114$.

34 Mengel-From J, Borsting C, Sanchez JJ, Eiberg H, Morling N: Human eye colour and HERC2, OCA2 and MATP. Forensic Sci Int Genet 2010; 4: 323-328.

35 R Development Core Team R: A Language and Environment for Statistical Computing. Vienna, Austria: R Foundation for Statistical Computing, 2009.

36 Warnes G, Gorjanc G, Leisch F, Man M genetics: Population Genetics. R package version 1.3.4 2008 .

37 Venables WN, Ripley BD: Modern Applied Statistics with S4th edn.New York: Springer, 2002.

38 Werft W, Benner A, Potter DM glmperm: Inference in Generalized Linear Models. $\mathrm{R}$ package version 1.0-5 2013.
39 Brasil P DiagnosisMed: Diagnostic test accuracy evaluation for medical professionals. $\mathrm{R}$ package version 0.2.3 2010 .

40 Robin X, Turck N, Hainard A et al: pROC: an open-source package for R and S+ to analyze and compare ROC curves. BMC Bioinformatics 2011; 12: 77.

41 So HC, Gui AH, Cherny SS, Sham PC: Evaluating the heritability explained by known susceptibility variants: a survey of ten complex diseases. Genet Epidemiol 2011; 35: 310-317

42 Faul F, Erdfelder E, Buchner A, Lang A-G. Statistical power analyses using G*Power 3.1: tests for correlation and regression analyses. Behav Res Methods 2009; 41: 1149-1160.

43 Novembre J, Johnson T, Bryc K et al: Genes mirror geography within Europe. Nature 2008; 456: 98-101.

44 Lao O, Lu TT, Nothnagel M et al: Correlation between genetic and geographic structure in Europe. Curr Biol 2008; 18: 1241-1248.

45 Steffens M, Lamina C, Illig T et al: SNP-based analysis of genetic substructure in the German population. Hum Hered 2006; 62: 20-29.

46 Walsh S, Wollstein A, Liu F et al: DNA-based eye colour prediction across Europe with the IrisPlex system. Forensic Sci Int Genet 2012; 6: 330-340.

47 Liu F, van Duijn K, Vingerling JR et al: Eye color and the prediction of complex phenotypes from genotypes. Curr Biol 2009; 19: R192-R193.

48 Pneuman A, Budimlija ZM, Caragine T, Prinz M, Wurmbach E: Verification of eye and skin color predictors in various populations. Leg Med (Tokyo) 2012; 14: 78-83.

49 Ruiz Y, Phillips C, Gomez-Tato A et al: Further development of forensic eye color predictive tests. Forensic Sci Int Genet 2013; 7: 28-40.

50 Walsh S, Liu F, Wollstein A et al: The HIrisPlex system for simultaneous prediction of hair and eye colour from DNA. Forensic Sci Int Genet 2013; 7: 98-115.

51 Cook NR: Use and misuse of the receiver operating characteristic curve in risk prediction. Circulation 2007; 115: 928-935.

52 Hand DJ, Anagnostopoulos C: When is the area under the receiver operating characteristic curve an appropriate measure of classifier performance? Pattern Recogn Lett 2013; 34: 492-495.

53 Lu TT, Lao O, Nothnagel M et al: An evaluation of the genetic-matched pair study design using genome-wide SNP data from the European population. Eur J Hum Genet 2009; 17: 967-975.

Supplementary Information accompanies this paper on European Journal of Human Genetics website (http://www.nature.com/ejhg) 